\title{
DESARROLLO DE SISTEMA WEB PARA VENTA DE LIBROS POR INTERNET EN LA BIBLIOTECA «ING. CÉSAR O. PALACIO TAPIA DE LA UJAT». USANDO LAS REDES SOCIALES
}

\author{
Abel del Carmen Jiménez Fuentes*,,José Juan Galicia Hernández *, Carlos Mario Flores Lázaro **, José Jaime \\ Ronzón Contreras **, Ninfa Urania García Ulin**, Gilberto Murillo González **
}

\begin{abstract}
Jiménez-Fuentes A.C , Galicia-Hernández J.J., FloresLázaro C.M., Ronzón-Contreras J.J., García-Ulin N.U., Murillo-González G. Desarrollo de sistema WEB para venta de libros por internet en la biblioteca «Ing. César O. Palacio Tapia de la ujat». Usando las redes sociales. Hitos de Ciencias Económico Administrativas 2016; 22 (62): 37-44
\end{abstract}

\section{RESUMEN}

La Biblioteca «Ing. César O. Palacio Tapia» de la Universidad Juárez Autónoma de Tabasco, no cuenta con un Sistema Web para la venta de libros, como un servicio más que permita a los usuarios comprar por Internet. Surgió un proyecto para vender libros en línea, mediante un Sistema Web, acatando necesidades de estudiantes, maestros y público en general. El objetivo de este proyecto es enfocarse al comercio electrónico, dado que es un medio conocido mundialmente por los usuarios. La elaboración de este trabajo se realizó con la herramienta WYSIWYG Web Builder. Este servicio funciona mediante una conexión que inicia desde la Biblioteca Campus Cunduacán «Ing. César O. Palacio Tapia», hacia la Librería Universitaria de la UJAT, ubicada en Villahermosa, Tabasco. Al sistema se podrá acceder desde cualquier terminal, a disposición del comprador. El punto de venta es la Biblioteca Campus Cunduacán. Este proyecto favorece a alumnos, profesores y público en general que dedican su tiempo libre a la lectura.

Palabras clave: Sistema Web. Comercio Electrónico.
Jiménez-Fuentes A.C , Galicia-Hernández J.J., FloresLázaro C.M., Ronzón-Contreras J.J., García-Ulin N.U., Murillo-González G. Desarrollo de sistema WEB para venta de libros por internet en la biblioteca "Ing. César O. Palacio Tapia de la ujat». Usando las redes sociales. Hitos de Ciencias Económico Administrativas 2016; 22 (62): 37-44

\section{ABSTRACT}

The Library «Ing. Cesar o. Palacio Tapia « from «Universidad Juarez Autonoma de Tabasco», does not have a Web System for selling books as a service that allows users to shop online. It emerged a project to sell books online, through a Web System, accepting needs of students, teachers and the general public. The objective of this project is to focus on electronic commerce, since it is a means known worldwide for users. The development of this work was done with the WYSIWYG Web Builder tool. This service works by initiating a connection from the Campus Library Cunduacán «»Ing. Cesar o. Palacio Tapia «from «Universidad Juarez Autonoma de Tabasco» to the University Bookstore UJAT, located in Villahermosa, Tabasco. System can be accessed from any terminal, to the buyer. The selling point is the Cunduacán Campus Library. This project encourages students, teachers and the general public who devote their free time to reading.

Key words: Web System. E-commerce.

DIRECCIÓN PARA RECIBIR CORRESPONDENCIA: Correo electrónico: jronzon@hotmail.com

\footnotetext{
"Licenciados en Sistemas Computacionales. Egresados de la División Académica de Informática y Sistemas (DAIS), de la Universidad Juárez Autónoma de Tabasco (UJAT).

" Profesores Investigadores de la DAIS-UJAT.
}

Fecha de recibido: 10 de noviembre de 2015 Fecha de aceptación: 7 de diciembre de 2015. 
$\mathbf{E}$

n el Municipio de Cunduacán, Tabasco México, se encuentra la segunda más grande de las Bibliotecas de la UJAT, por lo que asisten diariamente muchos estudiantes a sus instalaciones. Para más satisfacción la Biblioteca «Ing. César O. Palacio Tapia», propuso vender libros en línea mediante un sistema de venta de las diferentes editoriales incluyendo la editorial UJAT, para saber más a fondo que tan interesados están estos usuarios por este proyecto se aplicó un cuestionario de 20 preguntas, y parece ser que el $93 \%$ de 250 encuestados están de acuerdo que se lleve a cabo este afortunado trabajo. Por consiguiente, se determinó que muchos de los estudiantes si requieren de este servicio.

Como el proyecto es indispensable para los estudiantes y usuarios, se trabajó en el Sistema Web para vender libros en línea, que inicia de la Biblioteca Cunduacán a la Librería Universitaria de la UJAT, este trabajo es ofrecido más que nada a los estudiantes, maestros y público en general, y la Biblioteca «Ing. César O. Palacio Tapia» de la UJAT es la prestigiada para ofrecer este servicio. Para cubrir el objetivo se utilizó la herramienta WYSIWYG Web Builder, ya que es un software que cuenta con las características necesarias para el diseño y desarrollo de este Sistema Web, y además incluye la plataforma HTML5 que de igual manera trabaja para elaborar páginas Web.

\section{OBJETIVO GENERAL}

Desarrollar un prototipo de Sistema Web, para la venta de libros de la Biblioteca «Ing. César O. Palacio Tapia» de la UJAT, mediante la instalación de una terminal, que permita a los usuarios finales la compra de libros de la Editorial UJAT, como una extensión más del servicio de la Librería Universitaria.

\section{METAS}

Desarrollo del prototipo de Sistema Web, en base al autor Pressman, R. (2010: 457), las cuales son: prueba de contenido, prueba de interfaz, prueba de navegación y prueba de componentes.

\section{MARCO TEÓRICO}

\section{Sistemas Web}

Los sistemas Web, son aquellos que son desarrollados no sobre una plataforma o sistema operativo, sino que se montan en un servidor sobre una Intranet o Internet con un diseño similar al de una página Web, pero con capacidades superiores a estas. (http://www.xinapsys.com/ sistemasweb.aspx)

\section{Comercio Electrónico}

El comercio electrónico es definido por los estudios de la Organización para la Cooperación y el Desarrollo Económicos (OCDE), como el proceso de compra, venta o intercambio de bienes, servicios e información a través de las redes de comunicación. (www.profeco.gob.mx/ internacionales/com_elec.asp)

\section{MATERIAL Y MÉTODO}

\section{Población y muestra}

Para el desarrollo de la detección de las necesidades por parte de los alumnos del Campus Cunduacán, para ello se entrevistó al Director de la Biblioteca «Ing. César O. Palacio Tapia» de la UJAT, Lic. Andrés Alejandro Méndez Coello, el cual proporcionó la cifra de 650 alumnos que en promedio asisten diariamente a las instalaciones, tomando en cuenta la capacidad con la que se dispone y por la actividad de préstamos que se realizan durante el día, ello permitió el diseño de la muestra. 
Citando a Hernández, R; Fernández, C y Baptista, P. (1991: 266), señalan que «Cuando se hace una muestra probabilística, uno debe preguntarse ¿Cuál es el número mínimo de unidades de análisis (personas, organizaciones, capítulos de telenovelas, etc.), que necesito para conformar una muestra (n) que me asegure un error estándar menor de 0.01 (fijado por nosotros), dado que la población $\mathrm{N}$ es aproximadamente de tantos elementos? La fórmula para determinar el tamaño de $n$ es la siguiente»:

$$
n^{\prime}=\frac{s^{z^{\prime}}}{V^{2}} \frac{\text { Varianza de ia mucestra }}{\text { Varianza de la pos̀iación }}
$$

Lo cual se ajusta si se conoce el tamaño de la población $\mathrm{N}$. Entonces se tiene que:

$$
n=\frac{n^{\prime}}{1+n^{\prime} / N}
$$

Citando a Hernández, R; Fernández, C y Baptista, P. (1991: 266).

Tamaño provisional de la muestra:

$$
n^{\prime}=\frac{s^{z^{\prime}}}{v^{2}}
$$

Tamaño de la muestra:

$$
n=\frac{n^{\prime}}{1+n^{\prime} / N}
$$

Dado que:

$\mathrm{n}=$ La muestra.

$\mathrm{N}$ = Población de 650 individuos objetos de estudio.

$\mathrm{Se}=$ Error estándar $=.015$

$V^{2}=$ Varianza de la población.

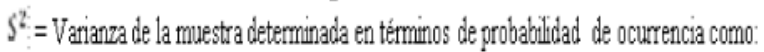
Sustituyendo las formulas, se obtienen los siguientes resultados:

$p(1-p)$, considerando un nivel de confianza del $90 \%$ el cual es el equivalente a 0.9
Por lo tanto:

$$
n=\frac{n^{r}}{1+n^{\prime} / k}=\frac{100}{1+100 / 650}=\frac{100}{1+0.615}=\frac{100}{1.615}=248
$$

Por lo que el tamaño de la muestra es de 248 individuos que asisten a la biblioteca.

Los instrumentos de recolección de datos que se implementaron fueron: la observación personal con respecto a un cartel tamaño tabloide, el cual contenía información para el desarrollo de esta investigación. Además, se realizó la aplicación de cuestionarios con preguntas cerradas, a los 248 estudiantes que asisten a la Biblioteca «Ing. César O. Palacio Tapia", por lo consiguiente se obtuvo información sobre el número de alumnos que son potencialmente compradores de libros.

Se observó a los estudiantes que asisten a las instalaciones de la Biblioteca «Ing. César $O$. Palacio Tapia» durante el trascurso de la semana y se recolectaron datos de acuerdo al interés que mostraban al ver el cartel. Por otra parte se aplicó un cuestionario, con preguntas concretas a los 248 individuos determinado anteriormente, en el lapso de una semana. Estos métodos de medición, se aplicaron, con el fin, de estudiar las tendencias de los usuarios de la Biblioteca, para el desarrollo de este proyecto.

Por otra parte, la prueba de un Sistema Web es un conjunto de actividades ordenadas con la finalidad, de descubrir errores en todo el contenido, función, utilidad, navegabilidad, rendimiento, capacidad y seguridad de dicho sistema. Para lograr esto, se aplica una prueba estratégica que abarca las revisiones, tanto como las pruebas ejecutables.

El autor Pressman, R. (2010: 457), maneja un proceso de prueba como puede apreciarse en la Figura 1, la cual se tomó como prueba para este proyecto. Los principales puntos a evaluar son los siguientes: prueba de contenido, prueba de interfaz, prueba de navegación, prueba de componente. 


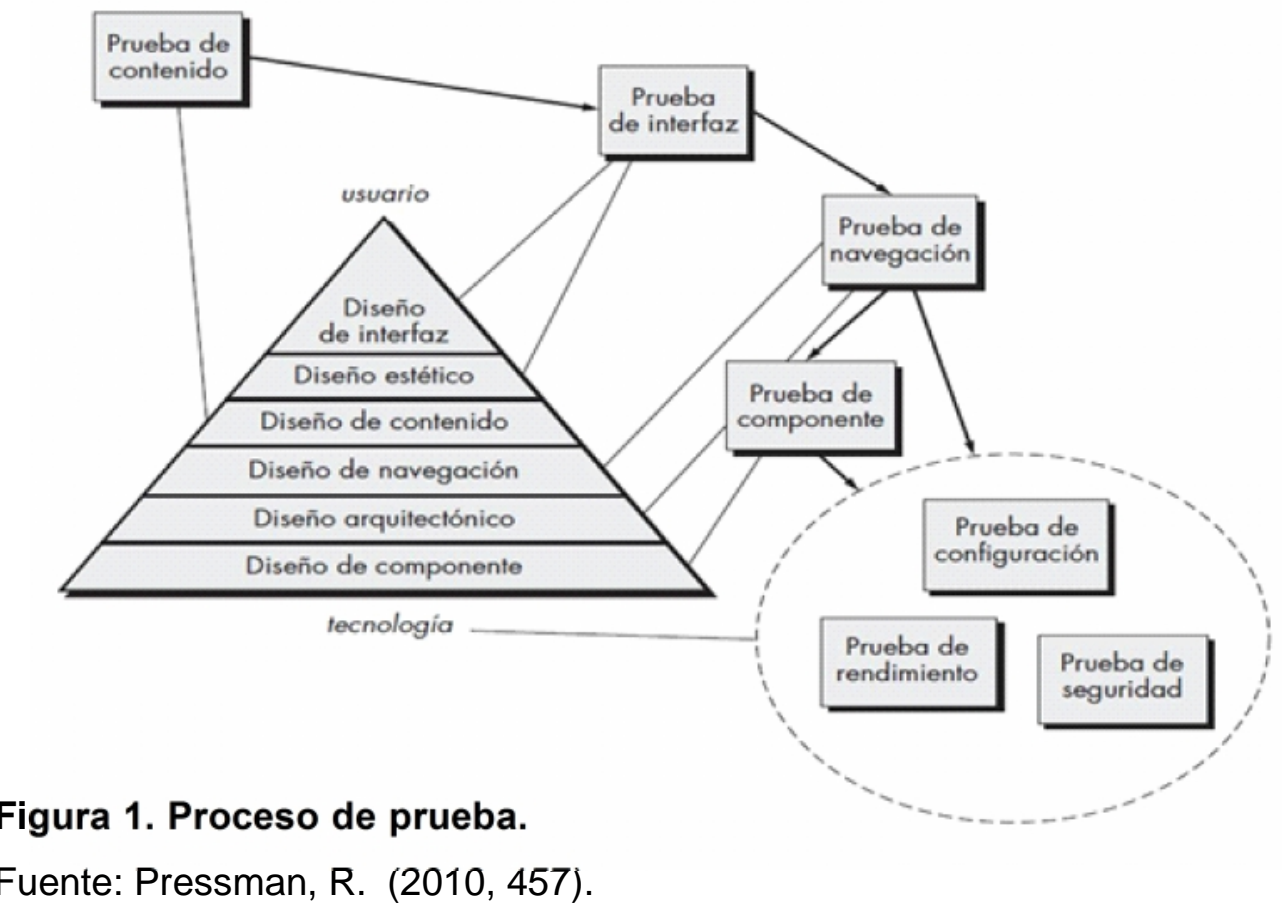

\section{Prueba de contenido}

«La prueba de contenido tiene tres objetivos importantes: 1) descubrir errores sintácticos (por ejemplo, errores tipográficos o gramaticales) en documentos de texto, representaciones gráficas y otros medios; 2) descubrir errores semánticos (es decir, errores en la precisión o completitud de la información) en cualquier objeto de contenido que se presente conforme ocurre la navegación y 3) encontrar errores en la organización o estructura del contenido que se presenta al usuario final». Pressman, R. (2010, 457).

\section{Prueba de interfaz}

«La prueba de interfaz ejercita los mecanismos de interacción y valida los aspectos estéticos de la interfaz de usuario. La estrategia global para la prueba de interfaz es: 1) descubrir errores relacionados con mecanismos de interfaz específicos (por ejemplo, en la ejecución adecuada de un vínculo de menú o en la forma como entran los datos en un formulario) y 2) descubrir errores en la forma como la interfaz implanta la semántica de navegación, la funcionalidad de la webapp o el despliegue de contenido». Pressman, R. (2010, 460).

\section{Prueba navegación}

«Los mecanismos de navegación se prueban para asegurarse de que cada interfaz realiza la función que se le ha encargado», Pressman, R. (2010, pp. 467-468).

\section{Prueba de componente}

«La prueba en el nivel de componente, también llamada prueba de función, se enfoca en un conjunto de pruebas que intentan descubrir errores en funciones de las webapps. Cada función de una webapp es un componente de software (implantado en uno de varios lenguajes de programación o lenguajes de guiones)», Pressman, R. (2010, 466).

"Los casos de prueba en el nivel de componente con frecuencia se derivan de la entrada a formularios. Una vez definidos los datos de los formularios, el usuario selecciona un botón u otro mecanismo de control para iniciar la ejecución». Pressman, R. (2010, 466). 


\section{RESULTADOS}

Para este primer análisis se detalla la información recolectada por el instrumento cualitativo. El cual fue observar la conducta de interés hacia el cartel por parte de alumnos, profesor o cualquier persona que ingresara a las instalaciones de la Biblioteca «Ing. César O. Palacio Tapia», esta observación se realizó durante una semana, en un horario corrido de 08:00 a 17:00, ya que en este horario es donde hay más concurrencia, esto en base a la información proporcionada por el Director de la Biblioteca. Es importante recalcar que se observaron a un total de 2599 personas. A continuación, se muestra la tabla 1, con un $90 \%$ se muestra cero interés, es decir que no observaron ni por un instante el cartel, el 5\% muestra poco interés, mientras que un 4\% muestra mucho interés, dicho de otra manera, se detuvieron a leer la información que contenía el cartel.
Para este segundo análisis se detalla la información recolectada por medio del instrumento cuantitativo, cabe recalcar una vez más, que el universo de estudio es de 650 personas en promedio que asisten a las instalaciones de la Biblioteca «Ing. César O. Palacio Tapia». Del cual el tamaño de la muestra fue de 248, cifra que se elevó a 250 para el mejor manejo de la información.

A continuación, se presentan solo los reactivos más importantes.

La tabla 2, es el resultado a la pregunta número 18.- ¿Te gustaría que la Biblioteca «Ing. César $O$. Palacio Tapia» contara con el servicio de ventas de libros por Internet?

\section{Tabla 1.}

Información recolectada por el instrumento cualitativo

\begin{tabular}{|cccc|}
\hline Día & Variable & Porcentaje & $\begin{array}{c}\text { Cantidad de personas } \\
\text { observadas }\end{array}$ \\
\hline \multirow{2}{*}{$\mathbf{5}$ días } & Cero interés & $90 \%$ & 2345 \\
\cline { 2 - 4 } & Poco interés & $5 \%$ & 141 \\
\cline { 2 - 4 } & Mucho interés & $4 \%$ & 113 \\
\hline & & $\mathbf{2 5 9 9}$ \\
\hline
\end{tabular}

Fuente: Biblioteca «Ing. César O. Palacio Tapia».

Tabla No. 2

Información recolectada con respecto al reactivo No. 18.

\begin{tabular}{|lcc|}
\hline \multicolumn{1}{|c}{ Respuesta } & Porcentaje & Cantidad \\
\hline NO & $7 \%$ & 17 \\
\hline SI & $93 \%$ & 233 \\
\hline Total general & $\mathbf{1 0 0} \%$ & $\mathbf{2 5 0}$ \\
\hline
\end{tabular}

Fuente: Biblioteca «Ing. César O. Palacio Tapia». 
En la tabla 2, puede apreciarse claramente que el 93\% de todos los encuestados afirmaron que si les gustaría que la Biblioteca «Ing. César O. Palacio Tapia», contara con el servicio de ventas de libros por Internet, mientras que un 7\% afirmó que no les gustaría. Es muy claro que la mayoría está de acuerdo en que se implemente este servicio.

La tabla 3, es el resultado a la pregunta número 19.- ¿Indica que editoriales te gustaría que estuvieran disponibles?

\section{Tabla}

Información recolectada con respecto al reactivo No. 19

\begin{tabular}{|lll|}
\hline Respuesta & Porcentaje & Cantidad \\
\hline A) Editorial UJAT & $16 \%$ & 83 \\
\hline B) Trillas & $18 \%$ & 96 \\
\hline C) Alfay Omega & $14 \%$ & 75 \\
\hline D) McGraw-Hill & $27 \%$ & 145 \\
\hline E) Pearson & $14 \%$ & 72 \\
\hline $\begin{array}{l}\text { F) Thompson } \\
\text { Learning }\end{array}$ & $10 \%$ & 53 \\
\hline Limusa & $0.2 \%$ & 1 \\
\hline Diana & $0.2 \%$ & 1 \\
\hline Gustavo Gill & $0.4 \%$ & 2 \\
\hline Total general & $100 \%$ & 528 \\
\hline
\end{tabular}

Fuente: Biblioteca «Ing. César O. Palacio Tapia».

En la Tabla No. 3 puede observarse que el total es de 528, cuando el tamaño de la muestra es de 250, eso se debe a que en el reactivo No. 19, una persona puede elegir varias opciones, por tal motivo se contabilizó las respuestas por cada inciso, además podían sugerir editoriales. Se realizó el ajuste para que el resultado del porcentaje fuera del $100 \%$.

La Tabla No. 4, es el resultado a la pregunta número 20.- ¿Te gustaría que este servicio de ventas estuviera asociado con las Redes Sociales?

\begin{tabular}{|lcc|}
\hline \multicolumn{1}{|c}{ Respuestas } & Porcentaje & Cantidad \\
\hline SI & $86 \%$ & 34 \\
\hline NO & $14 \%$ & 216 \\
\hline Total general & $\mathbf{1 0 0 \%}$ & $\mathbf{2 5 0}$ \\
\hline
\end{tabular}

De acuerdo con la tabla 4 , el $86 \%$ del total de los encuestados, afirmó que si están de acuerdo a que el servicio de ventas este asociado con la redes sociales, mientras el $14 \%$ no está de acuerdo.

Aquí se menciona los resultados de las pruebas que se efectuaron en las instalaciones de la Biblioteca «Ing. César O. Palacio Tapia», con la ayuda de un grupo de estudiantes que asisten diariamente. Ellos manipularon el Sistema Web en base a las instrucciones de un sencillo cuestionario con las preguntas adecuadas para saber el funcionamiento del Sistema.

Tomando en cuenta el proceso de prueba del autor Pressman, R. (2010, 457), se llegó al siguiente resultado:

El resultado de la prueba de contenido fue muy satisfactorio, los usuarios no encontraron errores gramaticales, errores semánticos o errores de estructura. Agregaron que la información era bastante clara, podían identificar cada aspecto del Sistema Web.

El resultado de la prueba de interfaz, es aceptada por los usuarios, ya que es similar al sitio oficial de la UJAT, el 
desglose del menú en la opción del catálogo, les resultó muy agradable y de fácil identificación con cada uno de ello. El manejo de la compra, puede resultar un tanto complicada, hay opciones de ayuda para que el usuario pueda informarse y conozca el procedimiento que debe de seguir al momento de proporcionar sus datos. Los usuarios notaron e identificaron con claridad cada vínculo con respecto a los textos normales del Sistema web.

Los resultados de pruebas de navegación, fueron satisfactorios, no encontraron dificultad al acceder a los vínculos de navegación, a la redirección, regresar a la página principal y el buscador interno del sistema Web.

El resultado de la prueba de componente con base al tiempo que midieron los usuarios fue muy corto, nada desesperante. El tiempo de respuesta fue muy rápido.

Las pruebas realizadas fueron un éxito, se demostró que los usuarios no presentan dificultades para interactuar con el sistema Web, la información es muy clara y ordenada, es fácil identificar los vínculos, acceder al menú desplegable y sobre todo es muy sencilla de utilizar.

\section{DISCUSIONES}

Se desarrolló satisfactoriamente un prototipo de Sistema Web, para la venta de Libros de la Biblioteca «Ing. César O. Palacio Tapia» de la UJAT, como resultado de la metodología aplicada, logrando cumplir las expectativas expuestas en el objetivo general. Se tomó en cuenta el estándar que maneja la UJAT, para hacer más cómoda y fácil la manipulación del prototipo de sistema Web.
En base a todos los estudios realizados anteriormente, se pudieron dar contestación a dos incógnitas:

¿Cuál es el impacto que tuvo el desarrollo de esta aplicación de los diferentes usuarios de la Biblioteca «Ing. César O. Palacio Tapia» de la UJAT, de tal forma que permita la compra de libros de estos usuarios, ante la Librería Universitaria?

El impacto fue alto, y la aceptación por parte de los usuarios para que este proyecto de investigación se implemente en un futuro fue de un $93 \%$ del total de todos los encuestados; es decir, que más de la mayoría está de acuerdo en este proyecto, que traerá consigo beneficios tanto para la institución, como para los que albergan en ella.

¿Quiénes son los usuarios que desearan comprar los libros?

Todas las personas mayores de edad son usuarios, que tengan acceso a tarjetas de crédito o débito, son potencialmente compradores de libros o de cualquier otro servicio que se ofrezca el Sistema Web. Al brindar este servicio en una Institución tan reconocida, ofrecerá mucha confianza y seguridad.

El Sistema Web vinculado en las redes sociales como motor de publicidad, fue aceptado por el $86 \%$ de los encuestados, concordando que sería bueno que estuviese ligado a las mismas. Cabe destacar, que muchas personas con acceso a internet a través de un dispositivo, visita las redes sociales más famosas, tal es el caso de Facebook y Twitter.

Se superaron los objetivos al ofrecer un Sistema Web totalmente estandarizado al sitio oficial de la UJAT, logrando con ella la rápida identificación del menú, el cual contiene un desglose del catálogo, con distintas ramas de estudio. Por otra parte, se logró con éxito manejar la venta de libros 
a través de Internet de una manera rápida y segura.

Fue incluido además la opción de contacto, que servirá para que los usuarios puedan solicitar un material que no esté incluido en el catálogo.

REFERENCIAS

Hernández, R; Fernández, C y Baptista, P. (1991: 255-266). Metodología de la Investigación. Primera Edición. México: McGraw-Hill.

Pressman, R. (2010: 457). Ingeniería de Software. Un Enfoque Práctico. (7a ed.). México: McGraw-Hill de México.

Referencias electrónicas

Comercio Electrónico. Recuperado de www.profeco.gob. $m x /$ internacionales/ com elec.asp

Sistema Web. Recuperado de http:// www.xinapsys.com/sistemasweb.aspx) 